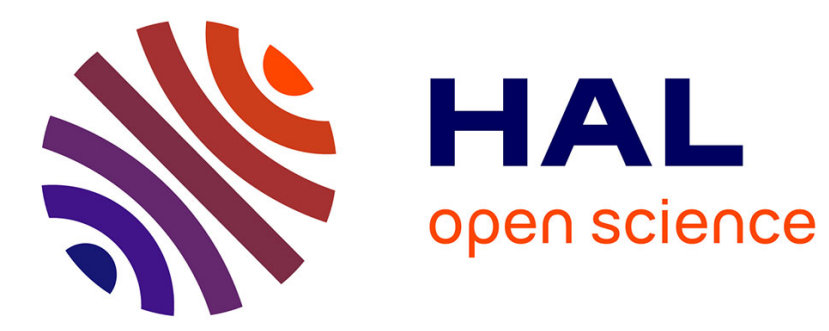

\title{
With exhaustible resources, can a developing country escape from the poverty trap?
}

Cuong Le Van, Katheline Schubert, Tu-Anh Nguyen

\section{To cite this version:}

Cuong Le Van, Katheline Schubert, Tu-Anh Nguyen. With exhaustible resources, can a developing country escape from the poverty trap?. 2007. halshs-00203180

\section{HAL Id: halshs-00203180 \\ https://shs.hal.science/halshs-00203180}

Submitted on 9 Jan 2008

HAL is a multi-disciplinary open access archive for the deposit and dissemination of scientific research documents, whether they are published or not. The documents may come from teaching and research institutions in France or abroad, or from public or private research centers.
L'archive ouverte pluridisciplinaire HAL, est destinée au dépôt et à la diffusion de documents scientifiques de niveau recherche, publiés ou non, émanant des établissements d'enseignement et de recherche français ou étrangers, des laboratoires publics ou privés. 


\section{Documents de Travail du Centre d'Economie de la Sorbonne}

.

$\mathrm{E}$

N

W

0

1

K

i

n

g

P

a

$\mathrm{p}$

e

r

$\mathrm{S}$

Cuong LE VAN, Katheline Schubert, Tu Anh NGUYeN

2007.75 


\title{
With Exhaustible Resources, Can A Developing Country Escape From The Poverty Trap? *
}

\author{
Cuong Le Van ${ }^{+\dagger}$ Katheline Schubert ${ }^{+}$and Tu Anh Nguyen ${ }^{++}$ \\ + Université Paris 1 Panthéon-Sorbonne, CNRS, Paris School of Economics \\ ++ Université Paris 1 Panthéon-Sorbonne, CNRS \\ CES, 106-112 bd de l' Hôpital, 75647 Paris Cedex 13, France.
}

July 2, 2007

\begin{abstract}
This paper studies the optimal growth of a developing non-renewable natural resource producer, which extracts the resource from its soil, and produces a single consumption good with man-made capital. Moreover, it can sell the extracted resource abroad and use the revenues to buy an imported good, which is a perfect substitute of the domestic consumption good. The domestic technology is convex-concave, so that the economy may be locked into a poverty trap. We study the optimal extraction and depletion of the exhaustible resource, and the optimal paths of accumulation of capital and of domestic consumption. We show that the extent to which the country will optimally escape from the poverty trap and the exhaustible resource will be a blessing depends on the characteristics of its technology and of the revenues from the resource function, on its impatience, on the level of its initial stock of capital, and on the abundance of the natural resource. If the marginal productivity of capital at the origin is greater than the sum of the social discount rate and the depreciation rate, the country will accumulate capital along the entire growth path, and will escape from the poverty trap, whatever its initial stocks of capital and resource, and provided that the marginal revenue obtained from the exportation of the resource is finite at the origin. On the contrary, if the marginal productivity of capital is lower than the depreciation rate whatever the level of capital, and if moreover the initial stock of capital is small, then the country will never accumulate; it will consume the revenues obtained from selling abroad the extracted resource, until there is

*E-mail: levan@univ-paris1.fr, schubert@univ-paris1.fr, nguyen.heraclex@gmail.com

${ }^{\dagger}$ Corresponding author
\end{abstract}


no resource left and the economy collapses. We also show that any optimal path may be decentralized in a competitive equilibrium by using a tax/subsidy scheme for firms.

Keywords: optimal growth, exhaustible resource, convex-concave technology, poverty trap, competitive equilibrium with tax/subsidy.

JEL Classification: Q32, C61 


\section{Introduction}

The standard literature on growth and exhaustible resources, initiated by Dasgupta and Heal [2] in the seventies, deals with developed economies, or a world economy, relying on a non-renewable natural resource as a factor of production. Capital and resource are imperfect substitutes in the production process. The resource input is necessary in the sense that there is no production without it, but unessential in the sense that its productivity at the origin is unbounded. When the social planner adopts a social welfare function of the discounted utilitarian type, the shadow price of the resource stock follows the Hotelling's rule, the resource is asymptotically depleted, and consumption asymptotically vanishes.

The problematic of this paper is different. We are concerned here with a developing non-renewable natural resource producer -an oil producing country for instance-, which extracts the resource from its soil in its primary sector, and produces a single consumption good with man-made capital in its secondary sector. Moreover, it can sell the extracted resource abroad ${ }^{1}$. The revenues are then used to buy an imported good, which is a perfect substitute of the domestic consumption good. The resource is unnecessary in the preceeding sense: domestic production is possible without it.

The question we want to address is the following: Can the ownership of non-renewable natural resources allow a poor country to make the transition out of a poverty trap? We suppose that the production function is convex for low levels of capital and concave for high levels. The conditions of occurrence of a poverty trap are then fulfilled (Dechert and Nishimura [3], Azariadis and Stachurski [1]): the country, if initially poor, may be unable to pass beyond the trap level of capital, that is to say to develop. But the country can also extract its resource, sell it abroad, and use the revenues to import the good. The natural resource is a source of income, which, together with the income coming from domestic production, can be used to consume, or to accumulate capital. The idea is that a poor country with abundant natural resources could extract and sell an amount of resource which would enable it to accumulate a stock of capital sufficient to overcome the weakness of its initial stock. We want to know on what circumstances would such a scenario optimally occur.

We study in this paper the optimal extraction and depletion of the exhaustible resource, and the optimal paths of accumulation of capital and of domestic consumption. We take into account the characteristics of the domes-

\footnotetext{
${ }^{1}$ In the same spirit, Eliasson and Turnowsky [4] study the growth of a small economy exporting a renewable resource to import consumption goods, with a reference to fish for Iceland, or forestry products for New-Zealand.
} 
tic technology, the shape of the foreign demand for the exhaustible resource, and of course the initial abundance of the resource and the initial level of development of the country.

We show that the extent to which the country will optimally escape from the poverty trap and the exhaustible resource will be a blessing depends on the characteristics of its technology and of the revenues from the resource function, on its impatience, on the level of its initial stock of capital, and on the abundance of natural resource. The technology is convex-concave, so a poverty trap exists. If the marginal productivity of capital at the origin is greater than the sum of the social discount rate and the depreciation rate, the country will accumulate capital along the entire growth path, and will escape from the poverty trap, whatever its initial stocks of capital and resource, and provided that the marginal revenue obtained from the exportation of the resource is finite at the origin. On the contrary, if the marginal productivity of capital is lower than the depreciation rate whatever the level of capital, and if moreover the initial stock of capital is small, then the country will never accumulate; it will consume the revenues obtained from selling abroad the extracted resource, until there is no resource left and the economy collapses.

Finally, we show that any optimal path may be decentralized in a competitive equilibrium. Due to the existence of increasing returns in production for low levels of capital, it is necessary to introduce a tax/subsidy scheme to ensure that the competitive equilibrium exists. This scheme is based upon the difference between the values of the input and output.

The remaining of the paper is organized as follows. Section 2 presents the model. Section 3 gives the properties of the optimal growth paths. We prove in section 4 the existence of a competitive equilibrium. Section 5 provides a summary of the main results and concludes.

\section{The model}

We consider a country which possesses a stock of a non-renewable natural resource $\bar{S}$. This resource is extracted at a rate $R_{t}$, and then sold abroad at a price $P_{t}$, in terms of the numeraire, which is the domestic single consumption good. The inverse demand function for the resource is $P\left(R_{t}\right)$. The revenue from the sale of the natural resource, $\phi\left(R_{t}\right)=P\left(R_{t}\right) R_{t}$, is used to buy a foreign good, which is supposed to be a perfect substitute of the domestic good, used for consumption and capital accumulation. The domestic production function is $F\left(k_{t}\right)$, supposed to be convex for low levels of capital and then concave. The depreciation rate is $\delta$. We define the function $f\left(k_{t}\right)=F\left(k_{t}\right)+(1-\delta) k_{t}$, and we shall, in the following, name it for simplicity the technology. We are interested 
in the optimal growth of this country which, if its initial capital is low, can be locked into a poverty trap (Dechert and Nishimura [3]). Will the revenues coming from the extraction of the natural resource allow it to escape from the poverty trap? Or, on the contrary, will the existence of the natural resource, which allows the country to consume without producing, destroy any incentive to accumulate?

Formally, we have to solve problem $(P)$

$$
\max \sum_{t=0}^{+\infty} \beta^{t} u\left(c_{t}\right), \beta \in(0,1)
$$

under the constraints

$$
\begin{aligned}
\forall t, c_{t} & \geq 0, k_{t} \geq 0, R_{t} \geq 0, \\
c_{t}+k_{t+1} & \leq f\left(k_{t}\right)+\phi\left(R_{t}\right), \\
\sum_{t=0}^{+\infty} R_{t} & \leq \bar{S} \\
\bar{S} & >0, k_{0} \geq 0 \text { are given. }
\end{aligned}
$$

We denote by $Z\left(k_{0}, \bar{S}\right)$ the Value-function of Problem $(P)$. We make the following assumptions:

H1 The utility function $u$ is strictly concave, strictly increasing, continuously differentiable in $R_{+}$, and satisfies $u(0)=0, u^{\prime}(0)=+\infty$.

H2 The production function $F$ is continuously differentiable in $R_{+}$, strictly increasing, strictly convex from 0 to $k_{I}$, strictly concave for $k \geq k_{I}$, and $F^{\prime}(+\infty)<\delta$. Moreover, it satisfies $F(0)=0$.

H3 The revenue function $\phi$ is continuously differentiable, concave, strictly increasing from 0 to $\widehat{R} \leq+\infty$, and strictly decreasing for $R>\widehat{R}$. It also satisfies $\phi(0)=0$.

Throughout this paper, an infinite sequence $\left(x_{t}\right)_{t=0, \ldots,+\infty}$ will be denoted by $\mathbf{x}$. An optimal solution to Problem $(P)$ will be denoted by $\left(\mathbf{c}^{*}, \mathbf{k}^{*}, \mathbf{R}^{*}\right)$. We say that the sequences $\mathbf{c}, \mathbf{k}, \mathbf{R}$ are feasible from $k_{0}$ if they satisfy the constraints:

$$
\begin{aligned}
\forall t, c_{t} & \geq 0, k_{t} \geq 0, R_{t} \geq 0 \\
c_{t}+k_{t+1} & \leq f\left(k_{t}\right)+\phi\left(R_{t}\right), \\
\sum_{t=0}^{+\infty} R_{t} & \leq \bar{S}, \text { and } k_{0} \text { is given. }
\end{aligned}
$$

Let $\Omega\left(k_{0}, \bar{S}\right)$ denote the set of $(\mathbf{k}, \mathbf{R})$ feasible from $k_{0}$ and $\bar{S}$, i.e.,

$$
\begin{gathered}
\forall t, 0 \leq k_{t+1} \leq f\left(k_{t}\right)+\phi\left(R_{t}\right), 0 \leq R_{t} \\
\sum_{t=0}^{+\infty} R_{t} \leq \bar{S}, k_{0} \geq 0 \text { is given. }
\end{gathered}
$$


We first list some preliminary results necessary for the main results of our paper.

Lemma 1 The Value-function $Z$ is continuous in $k_{0}$, given $\bar{S}$.

Proof: We first prove that the correspondence $\Omega$ is compact-valued and continuous in $k_{0}$, for the product topology, given $\bar{S}$.

To prove that $\Omega\left(k_{0}, \bar{S}\right)$ is compact, take a sequence $\left\{\mathbf{k}^{\mathbf{n}}, \mathbf{R}^{n}\right\}$ which converges to $\{\mathbf{k}, \mathbf{R}\}$ for the product topology. First, observe that for any feasible $\mathbf{k}$ we have

$$
\forall t, 0 \leq k_{t+1} \leq f\left(k_{t}\right)+\phi\left(R_{t}\right) \leq f\left(k_{t}\right)+\max \{\phi(\widehat{R}), \phi(\bar{S})\} .
$$

Therefore, $\mathbf{k}$ will be in a compact set for the product topology (see e.g. Le Van and Dana [5]). Second,

$$
\forall n, \forall t, 0 \leq k_{t+1}^{n} \leq f\left(k_{t}^{n}\right)+\phi\left(R_{t}^{n}\right)
$$

hence, by taking the limits we get

$$
\forall t, 0 \leq k_{t+1} \leq f\left(k_{t}\right)+\phi\left(R_{t}\right)
$$

We have proved that the set of feasible $\mathbf{k}$ is closed for the product topology. It is obvious that the set of feasible $\mathbf{R}$ belongs to a fixed compact set. To prove that this set is closed, observe that $\forall N, \forall n \sum_{t=0}^{N} R_{t}^{n} \leq \bar{S}$. Taking the limit we get $\forall N, \forall n \sum_{t=0}^{N} R_{t} \leq \bar{S}$. That implies $\sum_{t=0}^{+\infty} R_{t} \leq \bar{S}$. Summing up, we have proved that $\Omega\left(k_{0}, \bar{S}\right)$ is compact.

It is easy to check that $\Omega$ is upper hemi-continuous in $k_{0}$. It is less easy for the lower hemi-continuity of $\Omega$. We will prove that, actually, $\Omega$ is lower hemi-continuous. Let $k_{0}^{n} \rightarrow k_{0}$ as $n$ goes to $+\infty$ and $(\mathbf{k}, \mathbf{R}) \in \Omega\left(k_{0}, \bar{S}\right)$. We have to show there exists a subsequence still denoted by $\left(\mathbf{k}^{\mathbf{n}}, \mathbf{R}^{\mathbf{n}}\right)$, for short, which converges to $(\mathbf{k}, \mathbf{R})$ and satisfies $\left(\mathbf{k}^{\mathbf{n}}, \mathbf{R}^{\mathbf{n}}\right) \in \Omega\left(k_{0}^{n}, \bar{S}\right), \forall n$. We have three cases.

Case 1:

$$
\begin{aligned}
0 \leq k_{t+1} & <f\left(k_{t}\right)+\phi\left(R_{0}\right), \forall t<T-1 \\
0 \leq k_{t} & \leq f\left(k_{t-1}\right)+\phi\left(R_{t-1}\right), \forall t \geq T
\end{aligned}
$$

There exists $N$ such that for any $n \geq N$, we have $k_{1}<f\left(k_{0}^{n}\right)+\phi\left(R_{0}\right)$. Define, for any $n \geq N$, any $t, k_{t}^{n}=k_{t}, R_{t}^{n}=R_{t}$ and the proof is done.

Case 2:

$$
\begin{aligned}
k_{t+1} & =f\left(k_{t}\right)+\phi\left(R_{t}\right), \forall t \leq T-1, \\
k_{T+1} & <f\left(k_{T}\right)+\phi\left(R_{T}\right), \\
k_{t+1} & \leq f\left(k_{t}\right)+\phi\left(R_{t}\right), \forall t \geq T+1 .
\end{aligned}
$$


Define, for $t=0, \ldots, T-1$ and for any $n, k_{t+1}^{n}=f\left(k_{t}^{n}\right)+\phi\left(R_{t}\right)$. Obviously, $k_{t}^{n} \rightarrow k_{t}$ for $t=0, \ldots, T-1$. Hence, there exists $N$ such that for any $n \geq N$, $k_{T+1}<f\left(k_{T}^{n}\right)+\phi\left(R_{T}\right)$. The sequences $\left(k_{0}^{n}, k_{1}^{n}, \ldots, k_{T}^{n}, k_{T+1}, k_{T+2}, \ldots\right)$ and $\mathbf{R}^{\mathbf{n}}$ $=\mathbf{R}$, for every $n$, satisfy the required conditions.

Case 3:

$$
\forall t, k_{t+1}=f\left(k_{t}\right)+\phi\left(R_{t}\right) .
$$

It suffices to take $k_{t+1}^{n}=f\left(k_{t}^{n}\right)+\phi\left(R_{t}\right)$ for every $t$, every $n$.

The second step is to prove that the intertemporal utility function is continuous on the feasible set for the product topology. But the proof is standard (see e.g. Le Van and Dana [5]).

The third step is to apply the Maximum Theorem to conclude that $Z$ is continuous in $k_{0}$.

Lemma 2 There exists a constant $A$ which depends on $k_{0}, \widehat{R}$, and $\bar{S}$, such that for any feasible sequence $(\mathbf{c}, \mathbf{k}, \mathbf{R})$, we have $\forall t, 0 \leq c_{t} \leq A, 0 \leq k_{t} \leq A$.

Moreover, Problem (P) has an optimal solution. If $k_{I}=0$, then the solution is unique.

Proof: It is obvious that $R_{t} \leq \bar{S}, \forall t$. Now, if $\widehat{R}<+\infty$ then for any $t$, we have $c_{t}+k_{t+1} \leq f\left(k_{t}\right)+\phi(\hat{R})$. And if $\widehat{R}=+\infty$ then for all $t, c_{t}+k_{t+1} \leq$ $f\left(k_{t}\right)+\phi(\bar{S})$. Since $f^{\prime}(+\infty)<1$, from Le Van and Dana [5], page 17, there exists a constant $A$ which depends on $k_{0}, \hat{R}$ (if $\hat{R}<+\infty$ ) or on $k_{0}, \bar{S}$ such that $\forall t, 0 \leq c_{t} \leq A, 0 \leq k_{t} \leq A$.

We have already proved that the set of feasible sequences is compact for the product topology and the intertemporal utility function is continuous on the feasible set for the same topology. Hence, there exists a solution to Problem $(P)$. When $k_{I}$ equals 0 , because of the strict concavity of the technology and the utility function $u$, the solution will be unique.

\section{Properties of the optimal paths}

We now study the properties of the optimal paths.

In the following, the superscript ${ }^{*}$ denotes the optimal value of the variables.

Proposition 1 states that along the optimal path consumption is always strictly positive and the extraction always less than $\widehat{R}$, the maximum of the revenue function; moreover, if the marginal revenue is infinite when the extraction becomes very small, the resource will not be exhausted in finite time.

Proposition 1 For any $t, c_{t}^{*}>0$ and $R_{t}^{*}<\widehat{R}$. If $\phi^{\prime}(0)=+\infty$, then $R_{t}^{*}>0$ for all $t$. Obviously, $R_{t}^{*} \rightarrow 0$ as $t \rightarrow+\infty$. 
Proof: Let $V$ denote the Value-function. Observe that $V\left(k_{0}\right)>0$ for any $k_{0} \geq 0$, since the sequence $\mathbf{c}$ defined by $c_{0}=f\left(k_{0}\right)+\phi(\bar{S})$ and $c_{t}=0$ for any $t>0$ is feasible. Hence $V\left(k_{0}\right) \geq u\left(c_{0}\right)>0$. That implies $c_{t}^{*}>0, \forall t$, by the Inada condition $u^{\prime}(0)=+\infty$.

Let us prove that $R_{t}^{*}<\widehat{R}$ for all $t$. If $\widehat{R}=+\infty$, the proof is obvious. So, assume $\widehat{R}<+\infty$. We cannot have $R_{t}^{*}>\widehat{R}$ for some $t$, since $u$ is strictly increasing and $\phi$ is strictly decreasing for $R>\widehat{R}$. We cannot have $R_{t}^{*}=\widehat{R}$ for all $t$ since $\sum_{t=0}^{+\infty} R_{t}^{*}=\bar{S}$. If there exists $T$ with $R_{T}^{*}=\widehat{R}$, we can suppose $R_{T+1}^{*}<\widehat{R}$. Without loss of generality, take $T=0$. So

$$
\begin{aligned}
c_{0}^{*}+k_{1}^{*} & =f\left(k_{0}\right)+\phi(\widehat{R}) \\
c_{1}^{*}+k_{2}^{*} & =f\left(k_{1}^{*}\right)+\phi\left(R_{1}^{*}\right), \text { with } R_{1}^{*}<\widehat{R} .
\end{aligned}
$$

Choose $\varepsilon>0$ small enough such that $R_{1}^{*}+\varepsilon<\widehat{R}$ and $\widehat{R}-\varepsilon>0$. Let

$$
\begin{aligned}
c_{0}+k_{1}^{*} & =f\left(k_{0}\right)+\phi(\widehat{R}-\varepsilon) \\
c_{1}+k_{2}^{*} & =f\left(k_{1}^{*}\right)+\phi\left(R_{1}^{*}+\varepsilon\right) \\
\text { and } c_{t} & =c_{t}^{*}, \forall t \geq 2 .
\end{aligned}
$$

Let $\triangle_{\varepsilon}=\sum_{t=0}^{+\infty} \beta^{t} u\left(c_{t}\right)-\sum_{t=0}^{+\infty} \beta^{t} u\left(c_{t}^{*}\right)$. We have

$$
\begin{aligned}
\triangle_{\varepsilon} & =u\left(c_{0}\right)-u\left(c_{0}^{*}\right)+\beta\left[u\left(c_{1}\right)-u\left(c_{1}^{*}\right)\right] \\
& \geq u^{\prime}\left(c_{0}\right)\left[\phi^{\prime}(\widehat{R}-\varepsilon)(-\varepsilon)\right]+\beta u^{\prime}\left(c_{1}\right)\left[\phi^{\prime}\left(R_{1}^{*}+\varepsilon\right)(\varepsilon)\right] \\
& \geq \varepsilon\left[\beta u^{\prime}\left(c_{1}\right) \phi^{\prime}\left(R_{1}^{*}+\varepsilon\right)-u^{\prime}\left(c_{0}\right) \phi^{\prime}(\widehat{R}-\varepsilon)\right] .
\end{aligned}
$$

Let $\varepsilon \rightarrow 0$. Then $\lim _{\varepsilon \rightarrow 0} \triangle_{\varepsilon} \geq \beta u^{\prime}\left(c_{1}^{*}\right) \phi^{\prime}\left(R_{1}^{*}\right)>0$. Thus $\triangle_{\varepsilon}>0$ for $\varepsilon$ small enough. That is a contradiction to the optimality of $\mathbf{c}^{*}$.

Now consider the case $\phi^{\prime}(0)=+\infty$. First assume $R_{t}^{*}=0, \forall t$. Then let

$$
\begin{aligned}
& c_{0}=f\left(k_{0}\right)-k_{1}^{*}+\bar{S}>c_{0}^{*} \\
& c_{t}=f\left(k_{t}^{*}\right)-k_{t+1}^{*}=c_{t}^{*}, \text { for } t \geq 1 .
\end{aligned}
$$

Then $\sum_{t=0}^{+\infty} u\left(c_{t}\right)>\sum_{t=0}^{+\infty} u\left(c_{t}^{*}\right)$ : a contradiction. Hence if $R_{T}^{*}=0$ we can assume that $R_{T+1}^{*}>0$. Without loss of generality, take $T=0$. So

$$
\begin{aligned}
c_{0}^{*} & =f\left(k_{0}\right)-k_{1}^{*} \\
c_{1}^{*} & =f\left(k_{1}^{*}\right)-k_{2}^{*}+\phi\left(R_{1}^{*}\right), \text { with } 0<R_{1}^{*}<\widehat{R} .
\end{aligned}
$$

Let $\varepsilon \in\left(0, R_{1}^{*}\right)$. Define

$$
\begin{aligned}
& c_{0}=f\left(k_{0}\right)-k_{1}^{*}+\phi(\varepsilon) \\
& c_{1}=f\left(k_{1}^{*}\right)-k_{2}^{*}+\phi\left(R_{1}^{*}-\varepsilon\right) \\
& c_{t}=c_{t}^{*}, \forall t \geq 2 .
\end{aligned}
$$


Then

$$
\begin{aligned}
\triangle_{\varepsilon} & =\sum_{t=0}^{+\infty} \beta^{t} u\left(c_{t}\right)-\sum_{t=0}^{+\infty} \beta^{t} u\left(c_{t}^{*}\right) \\
& =u\left(c_{0}\right)-u\left(c_{0}^{*}\right)+\beta\left[u\left(c_{1}\right)-u\left(c_{1}^{*}\right)\right] \\
& \geq u^{\prime}\left(c_{0}\right) \phi(\varepsilon)+\beta u^{\prime}\left(c_{1}\right)\left[\phi\left(R_{1}^{*}-\varepsilon\right)-\phi\left(R_{1}^{*}\right)\right] \\
& \geq\left[u^{\prime}\left(c_{0}\right) \phi^{\prime}(\varepsilon)-\beta u^{\prime}\left(c_{1}\right) \phi^{\prime}\left(R_{1}^{*}-\varepsilon\right)\right] \varepsilon .
\end{aligned}
$$

Notice that $\lim _{\varepsilon \rightarrow 0} \frac{\Delta_{\varepsilon}}{\varepsilon}=+\infty$ which implies $\triangle_{\varepsilon}>0$ for $\varepsilon$ small enough: a contradiction.

Proposition 2 gives the Euler conditions of our problem, in the case where the marginal revenue at the origin is finite, which we will favor in the remaining of the paper. Notice that in the case of an interior solution, equations $(E 1)$ and (E2) allow us to obtain the Hotelling's rule:

$$
\frac{\phi^{\prime}\left(R_{t+1}^{*}\right)}{\phi^{\prime}\left(R_{t}^{*}\right)}=f^{\prime}\left(k_{t+1}^{*}\right)
$$

Proposition 2 Let $k_{0} \geq 0$. Assume $\phi^{\prime}(0)<+\infty$. Then we have the following Euler conditions:

$$
\text { (i) } \forall t, f^{\prime}\left(k_{t+1}^{*}\right) \leq \frac{u^{\prime}\left(c_{t}^{*}\right)}{\beta u^{\prime}\left(c_{t+1}^{*}\right)}
$$

with equality if $k_{t+1}^{*}>0$,

$$
\text { (ii) } \forall t, \forall t^{\prime}, \beta^{t} u^{\prime}\left(c_{t}^{*}\right) \phi^{\prime}\left(R_{t}^{*}\right)=\beta^{t^{\prime}} u^{\prime}\left(c_{t^{\prime}}^{*}\right) \phi^{\prime}\left(R_{t^{\prime}}^{*}\right),
$$

if $R_{t}^{*}>0, R_{t^{\prime}}^{*}>0$, and

$$
\text { (iii) } \forall t, \forall t^{\prime}, \beta^{t} u^{\prime}\left(c_{t}^{*}\right) \phi^{\prime}\left(R_{t}^{*}\right) \leq \beta^{t^{\prime}} u^{\prime}\left(c_{t^{\prime}}^{*}\right) \phi^{\prime}\left(R_{t^{\prime}}^{*}\right)
$$

if $R_{t}^{*}=0, R_{t^{\prime}}^{*}>0$.

Proof: (i) Given $t, k_{t+1}^{*}$ solves :

$$
\begin{aligned}
\max _{y} & {\left[u\left(f\left(k_{t}^{*}\right)+\phi\left(R_{t}^{*}\right)-y\right)+\beta u\left(f(y)+\phi\left(R_{t+1}^{*}\right)-k_{t+2}^{*}\right)\right] } \\
\text { s.t. } 0 \leq & y \leq f\left(k_{t}^{*}\right)+\phi\left(R_{t}^{*}\right) \\
0 \leq & y .
\end{aligned}
$$

Since $c_{t}^{*}=f\left(k_{t}^{*}\right)+\phi\left(R_{t}^{*}\right)-k_{t+1}^{*}>0$, one easily gets $(E 1)$. 
(ii) Since $\bar{S}>0$, there exists $t$ with $R_{t}^{*}>0$. Fix some $T$ such that there exists $t \leq T$ with $R_{t}^{*}>0$. Then $\left(R_{0}^{*}, \ldots, R_{T}^{*}\right)$ solve

$$
\begin{aligned}
\max _{\left(R_{0}, \ldots, R_{t}\right)} & \sum_{t=0}^{T} \beta^{t} u\left(f\left(k_{t}^{*}\right)+\phi\left(R_{t}\right)-k_{t+1}^{*}\right) \\
\text { s.t. } \sum_{t=0}^{T} R_{t} \leq & \bar{S}-\sum_{\tau=T+1}^{+\infty} R_{\tau}^{*} \\
0 & \leq R_{t}, \forall t=0, \ldots, T \\
k_{t+1}^{*}-f\left(k_{t}^{*}\right) & \leq \phi\left(R_{t}\right), \forall t=0, \ldots, T .
\end{aligned}
$$

Since $\phi$ is concave and $u$ is strictly concave, $\left(R_{0}^{*}, \ldots, R_{T}^{*}\right)$ will be the unique solution. Moreover, since $c_{t}^{*}>0$ for every $t$, the third constraints system will not be binding. There exist therefore $\lambda \geq 0$ and $\mu_{t} \geq 0, t=0, \ldots, T$ such that $\left(R_{0}^{*}, \ldots, R_{T}^{*}\right)$ maximize

$$
\sum_{t=0}^{T} \beta^{t} u\left(f\left(k_{t}^{*}\right)+\phi\left(R_{t}\right)-k_{t+1}^{*}\right)-\lambda\left[\sum_{t=0}^{T} R_{t}-\bar{S}+\sum_{\tau=T+1}^{+\infty} R_{\tau}^{*}\right]+\sum_{t=0}^{T} \mu_{t} R_{t},
$$

with $\mu_{t} R_{t}^{*}=0, \forall t=0, \ldots, T$. One easily obtains $(E 2)$ and $\left(E 2^{\prime}\right)$.

Proposition 3 shows that, even if the initial capital stock equals 0 , if the marginal productivity of capital at the origin is large enough, then, thanks to the exhaustible resource, the country will accumulate from some date on. More precisely, the marginal productivity at the origin of the production function $F$ must be larger than the depreciation rate $\delta$.

Proposition 3 Let $k_{0} \geq 0$. Assume $\phi^{\prime}(0)<+\infty$. If $f^{\prime}(0)>1$, then there exists $T \geq 1$ with $k_{t}^{*}>0$ for any $t \geq T$.

Proof: Since $f^{\prime}(0)>1$, we can choose $\epsilon>0$ such that $f^{\prime}(0)>1+\epsilon$. Assume that there exists an infinite sequence $\left\{k_{t^{\nu}}^{*}\right\}_{\nu}$ such that $k_{t^{\nu}}^{*}=0$, for any $\nu$, and hence correspondently $R_{t^{\nu}}^{*}>0$. Because $\sum_{t=o}^{+\infty} R_{t}^{*}=\bar{S}$ we have $R_{t^{\nu}}^{*} \longrightarrow 0$ as $\nu \longrightarrow+\infty$. Since $R_{t^{\nu}}^{*} \longrightarrow 0$ and $R_{t^{\nu}-1}^{*}$ either equals 0 or converges to 0 , there exists $T$ such that $\frac{\phi^{\prime}\left(R_{t^{\nu}}^{*}\right)}{\phi^{\prime}\left(R_{t^{\nu}-1}^{*}\right)}<1+\epsilon$ if $t^{\nu} \geq T$. We can write down the optimal consumptions at time $t^{\nu}$ and $t^{\nu}-1$ as follows:

$$
\begin{aligned}
c_{t^{\nu}-1}^{*} & =\phi\left(R_{t^{\nu}-1}^{*}\right)+f\left(k_{t^{\nu}-1}^{*}\right) \\
c_{t^{\nu}}^{*} & =\phi\left(R_{t^{\nu}}^{*}\right)-k_{t^{\nu}+1}^{*}
\end{aligned}
$$

We have

$$
\left.u\left(c_{t^{\nu}-1}^{*}-y\right)+\beta u\left(c_{t^{\nu}}^{*}+f(y)\right) \leq u\left(c_{t^{\nu}-1}^{*}\right)\right)+\beta u\left(c_{t^{\nu}}^{*}\right)
$$


for all $y \in\left[0, c_{t^{\nu}-1}^{*}\right]$, thus

$$
-u^{\prime}\left(c_{t^{\nu}-1}^{*}\right)+\beta u^{\prime}\left(c_{t^{\nu}}^{*}\right) f^{\prime}(0) \leq 0
$$

and we get a contradiction

$$
\begin{aligned}
1+\epsilon<f^{\prime}(0) & \leq \frac{u^{\prime}\left(c_{t^{\nu}-1}^{*}\right)}{\beta u^{\prime}\left(c_{t^{\nu}}^{*}\right)} \\
& \leq \frac{\phi^{\prime}\left(R_{t^{\nu}}^{*}\right)}{\phi^{\prime}\left(R_{t^{\nu}-1}^{*}\right)}<1+\epsilon .
\end{aligned}
$$

So, there must exist $T \geq 1$ such that $k_{t}^{*}>0$ for all $t \geq T$.

In Proposition 4, we show that thanks to the exhaustible resource, the country will accumulate at any period, provided that the marginal productivity of capital at the origin is larger than the sum of the social discount rate and the depreciation rate, $\rho+\delta$, with $\rho=\frac{1}{\beta}-1$. Notice that when the initial capital stock is equal to 0 , the same economy without natural resources never takes-off (Dechert and Nishimura [3]). So, when the technology is "good" enough, the natural resource is a blessing allowing the economy to escape from the poverty trap.

Proposition 4 Let $k_{0} \geq 0$. Assume $f^{\prime}(0)>\frac{1}{\beta}$. Then $k_{t}^{*}>0$ for any $t \geq 1$.

Proof: Assume $k_{1}^{*}=0$. Then we have

$$
\begin{aligned}
c_{0}^{*} & =f\left(k_{0}\right)+\phi\left(R_{0}^{*}\right) \\
c_{1}^{*}+k_{2}^{*} & =\phi\left(R_{1}^{*}\right) .
\end{aligned}
$$

The following Euler conditions hold:

$$
\begin{aligned}
-u^{\prime}\left(c_{0}^{*}\right)+\beta u^{\prime}\left(c_{1}^{*}\right) f^{\prime}(0) & \leq 0 \\
u^{\prime}\left(c_{0}^{*}\right) \phi^{\prime}\left(R_{0}^{*}\right)-\beta u^{\prime}\left(c_{1}^{*}\right) \phi^{\prime}\left(R_{1}^{*}\right) & \leq 0 .
\end{aligned}
$$

This implies

$$
1<\frac{1}{\beta}<f^{\prime}(0) \leq \frac{u^{\prime}\left(c_{0}^{*}\right)}{\beta u^{\prime}\left(c_{1}^{*}\right)} \leq \frac{\phi^{\prime}\left(R_{1}^{*}\right)}{\phi^{\prime}\left(R_{0}^{*}\right)} .
$$

¿From these inequalities, we get $u^{\prime}\left(c_{0}^{*}\right)>u^{\prime}\left(c_{1}^{*}\right), \phi^{\prime}\left(R_{1}^{*}\right)>\phi^{\prime}\left(R_{0}^{*}\right)$ or equivalently, $c_{1}^{*}>c_{0}^{*}$ and $R_{0}^{*}>R_{1}^{*}$. A contradiction arises:

$$
\phi\left(R_{1}^{*}\right) \geq \phi\left(R_{1}^{*}\right)-k_{2}^{*}=c_{1}^{*}>c_{0}^{*}=f\left(k_{0}\right)+\phi\left(R_{0}^{*}\right) \geq \phi\left(R_{0}^{*}\right)>\phi\left(R_{1}^{*}\right) .
$$

Therefore, $k_{1}^{*}>0$. By induction, $k_{t}^{*}>0$ for all $t \geq 1$.

We want to show now that the natural resource will be exhausted in finite time if the marginal productivity of capital at the origin is high enough. Before proving that, let us introduce an intermediary step. 
Consider Problem $(Q)$

$$
U\left(k_{0}\right)=\max \sum_{t=0}^{+\infty} \beta^{t} u\left(c_{t}\right), \beta \in(0,1)
$$

under the constraints

$$
\begin{aligned}
\forall t, c_{t} & \geq 0, k_{t} \geq 0, \\
c_{t}+k_{t+1} & \leq f\left(k_{t}\right), \\
k_{0} & \geq 0 \text { is given. }
\end{aligned}
$$

Let $\varphi$ denote the optimal correspondence of $(Q)$, i.e., $k_{1} \in \varphi\left(k_{0}\right)$ iff we have $k_{1} \in\left[0, f\left(k_{0}\right)\right]$ and

$$
\begin{aligned}
U\left(k_{0}\right) & =u\left(f\left(k_{0}\right)-k_{1}\right)+\beta U\left(k_{1}\right) \\
& =\max \left\{u\left(f\left(k_{0}\right)-y\right)+\beta U(y): y \in\left[0, f\left(k_{0}\right)\right]\right\} .
\end{aligned}
$$

Next consider Problem $\left(Q_{\mathbf{a}}\right)$ where $\mathbf{a}$ is a sequence of non-negative real numbers which satisfies $\sum_{t=0}^{+\infty} a_{t}<+\infty$ :

$$
W\left(k_{0},\left(a_{t}\right)_{t \geq 0}\right)=\max \sum_{t=0}^{+\infty} \beta^{t} u\left(c_{t}\right), \beta \in(0,1)
$$

under the constraints

$$
\begin{aligned}
\forall t, c_{t} & \geq 0, k_{t} \geq 0, \\
c_{t}+k_{t+1} & \leq f\left(k_{t}\right)+a_{t}, \\
k_{0} & \geq 0 \text { is given. }
\end{aligned}
$$

Obviously, $W\left(k_{0}, 0\right)=U\left(k_{0}\right)$, and $W\left(k_{0},\left(a_{t}\right)_{t \geq 0}\right) \geq U\left(k_{0}\right)$. We also have the Bellman equation: for all $k_{0}$,

$W\left(k_{0},\left(a_{t}\right)_{t \geq 0}\right)=\max \left\{u\left(f\left(k_{0}\right)-y+a_{0}\right)+\beta W\left(y,\left(a_{t}\right)_{t \geq 1}\right): y \in\left[0, f\left(k_{0}\right)+a_{0}\right]\right\}$

Let $\psi\left(.,\left(a_{t}\right)_{t \geq 0}\right)$ denote the optimal correspondence associated with $\left(Q_{\mathbf{a}}\right)$, i.e., $k_{1} \in \psi\left(k_{0},\left(a_{t}\right)_{t \geq 0}\right)$ iff $W\left(k_{0},\left(a_{t}\right)_{t \geq 0}\right)=u\left(f\left(k_{0}\right)-k_{1}+a_{0}\right)+\beta W\left(k_{1},\left(a_{t}\right)_{t \geq 1}\right)$ and $k_{1} \in\left[0, f\left(k_{0}\right)+a_{0}\right]$. We have the following lemma.

Lemma 3 Let $k_{0}^{n} \rightarrow k_{0}$ and $\mathbf{a}^{\mathbf{n}} \rightarrow \mathbf{0}$ in $l^{\infty}$ when $n$ converges to infinity. If, for any $n, k_{1}^{n} \in \psi\left(k_{0}^{n}, \mathbf{a}^{\mathbf{n}}\right)$ and $k_{1}^{n} \rightarrow k_{1}$ as $n \rightarrow+\infty$, then $k_{1} \in \varphi\left(k_{0}\right)$. 
Proof: We first prove that $W\left(k_{0}^{n}, \mathbf{a}^{\mathbf{n}}\right) \rightarrow U\left(k_{0}\right)$ as $n \rightarrow+\infty$. We have:

$$
\forall n, W\left(k_{0}^{n},\left(a_{t}^{n}\right)_{t \geq 0}\right) \geq U\left(k_{0}^{n}\right),
$$

hence

$$
\liminf _{n \rightarrow+\infty} W\left(k_{0}^{n},\left(a_{t}^{n}\right)_{t \geq 0}\right) \geq \lim _{n \rightarrow+\infty} U\left(k_{0}^{n}\right)=U\left(k_{0}\right) .
$$

We now prove that $\limsup _{n \rightarrow+\infty} W\left(k_{0}^{n},\left(a_{t}^{n}\right)_{t \geq 0}\right) \leq U\left(k_{0}\right)$. Let $\alpha>0$. There exists $N$ such that, for any $n \geq N$, we have $f\left(k_{0}^{n}\right)+a_{0}^{n} \leq f\left(k_{0}\right)+\alpha$ and $k_{0}^{n} \leq \alpha$. Let $\bar{k}^{\alpha}$ be the largest value of $k$ which satisfies $f\left(\bar{k}^{\alpha}\right)+\alpha=\bar{k}^{\alpha}$. Using the same argument as in Le Van and Dana [5], page 17, one can show that, for any feasible sequences from $k_{0}^{n}, \mathbf{c}^{\mathbf{n}}, \mathbf{k}^{\mathbf{n}}$ of $\left(Q_{\mathbf{a}^{\mathbf{n}}}\right)$, for any $n \geq N$, any $t$, we have $c_{t}^{n} \leq \max \left\{\bar{k}^{\alpha}, k_{0}+\alpha\right\}, k_{t}^{n} \leq \max \left\{\bar{k}^{\alpha}, k_{0}+\alpha\right\}$. Let $\mathbf{c}^{* \mathbf{n}}, \mathbf{k}^{* \mathbf{n}}$ be the optimal sequences from $k_{0}^{n}$ of Problem $\left(Q_{\mathbf{a}^{\mathbf{n}}}\right)$. Let $\varepsilon>0$. There exists $T$ such that

$$
\forall n, W\left(k_{0}^{n},\left(a_{t}^{n}\right)_{t \geq 0}\right) \leq \sum_{t=0}^{t=T} \beta^{t} u\left(c_{t}^{* n}\right)+\varepsilon
$$

For $t=0, \ldots T$, we can suppose that $c_{t}^{* n} \rightarrow \bar{c}_{t}$ and $k_{t+1}^{* n} \rightarrow \bar{k}_{t+1}$. Since for $t=0, \ldots T$, we have $c_{t}^{* n}+k_{t+1}^{* n}=f\left(k_{t}^{* n}\right)+a_{t}^{n}$, we obtain $\bar{c}_{t}+\bar{k}_{t+1}=f\left(\bar{k}_{t}\right)$ for $t=0, \ldots, T$. Define $\overline{\mathbf{c}}=\left(\bar{c}_{0}, \ldots, \bar{c}_{T}, 0,0, \ldots, 0, \ldots\right)$. We get

$$
\limsup _{n \rightarrow+\infty} W\left(k_{0}^{n},\left(a_{t}^{n}\right)_{t \geq 0}\right) \leq \sum_{t=0}^{t=T} \beta^{t} u\left(\bar{c}_{t}\right)+\varepsilon=\sum_{t=0}^{t=+\infty} \beta^{t} u\left(\bar{c}_{t}\right)+\varepsilon \leq U\left(k_{0}\right)+\varepsilon .
$$

This inequality holds for any $\varepsilon>0$. We have proved $\limsup _{n \rightarrow+\infty} W\left(k_{0}^{n}, \mathbf{a}^{\mathbf{n}}\right) \leq U\left(k_{0}\right)$.

Now, let $k_{1}^{n} \in \psi\left(k_{0}^{n}, \mathbf{a}^{\mathbf{n}}\right)$ and suppose $k_{1}^{n} \rightarrow k_{1}$ as $n \rightarrow+\infty$. We have

$$
W\left(k_{0}^{n},\left(a^{n}\right)_{t \geq 0}\right)=u\left(f\left(k_{0}^{n}\right)-k_{1}^{n}+a_{0}^{n}\right)+\beta W\left(k_{1}^{n},\left(a^{n}\right)_{t \geq 1}\right)
$$

and $k_{1}^{n} \in\left[0, f\left(k_{0}^{n}\right)+a_{0}^{n}\right]$. Taking the limits we get

$$
U\left(k_{0}\right)=u\left(f\left(k_{0}\right)-k_{1}\right)+\beta U\left(k_{1}\right),
$$

with $k_{1} \in\left[0, f\left(k_{0}\right)\right]$. That proves $k_{1} \in \varphi\left(k_{0}\right)$.

Proposition 5 states that if the marginal revenue of the resource at the origin is finite and the marginal productivity of capital greater than the depreciation rate, then the stock of resource will be exhausted in finite time.

Proposition 5 Assume $\phi^{\prime}(0)<+\infty$ and $f^{\prime}(0)>1$. Then there exists $T_{\infty}$ such that, for all $t \geq T_{\infty}$, we have $R_{t}^{*}=0$. 
Proof: ¿From Proposition 3, there exists $T$ such that $\forall t \geq T, k_{t}^{*}>0$.

Step 1. We will show that there exists $T^{\prime}$ such that $R_{T^{\prime}}^{*}=0$. If not, for any $t \geq T$ we have the Euler conditions:

$$
\begin{gathered}
\beta u^{\prime}\left(c_{t+1}^{*}\right) f^{\prime}\left(k_{t+1}^{*}\right)=u^{\prime}\left(c_{t}^{*}\right), \\
\beta u^{\prime}\left(c_{t+1}^{*}\right) \phi^{\prime}\left(R_{t+1}^{*}\right)=u^{\prime}\left(c_{t}^{*}\right) \phi^{\prime}\left(R_{t}^{*}\right) .
\end{gathered}
$$

Hence

$$
f^{\prime}\left(k_{t+1}^{*}\right)=\frac{u^{\prime}\left(c_{t}^{*}\right)}{\beta u^{\prime}\left(c_{t+1}^{*}\right)}=\frac{\phi^{\prime}\left(R_{t+1}^{*}\right)}{\phi^{\prime}\left(R_{t}^{*}\right)} .
$$

Since $\frac{\phi^{\prime}\left(R_{t+1}^{*}\right)}{\phi^{\prime}\left(R_{t}^{*}\right)} \rightarrow 1$, we have $f^{\prime}\left(k_{t+1}^{*}\right) \rightarrow 1$, as $t \rightarrow+\infty$. Under our assumptions there exists a unique $\widehat{k}$ which satisfies $f^{\prime}(\widehat{k})=1$. Thus $k_{t+1}^{*} \rightarrow \widehat{k}$. In this case, for $t$ large enough, $u^{\prime}\left(c_{t+1}^{*}\right)>u^{\prime}\left(c_{t}^{*}\right) \Leftrightarrow c_{t}^{*}>c_{t+1}^{*}$. The sequence $\mathbf{c}^{*}$ converges to $\bar{c}$. If $\bar{c}>0$, we have $f^{\prime}(\widehat{k})=\frac{1}{\beta}$ : a contradiction. So, $\bar{c}=0$. Since

$$
\forall t, c_{t+1}^{*}+k_{t+2}^{*}=f\left(k_{t+1}^{*}\right)+\phi\left(R_{t+1}^{*}\right),
$$

we have $\widehat{k}=f(\widehat{k})$ with $f^{\prime}(\widehat{k})=1$, and that is impossible. Hence, there must be $T^{\prime}$ with $R_{T^{\prime}}^{*}=0$.

Step 2. Assume there exists three sequences $\left(c_{t^{\nu}}^{*}\right)_{\nu},\left(k_{t^{\nu}}^{*}\right)_{\nu},\left(R_{t^{\nu}}^{*}\right)_{\nu}$ which satisfy

$$
\begin{aligned}
\forall \nu, c_{t^{\nu}-1}^{*}+k_{t^{\nu}}^{*} & =f\left(k_{t^{\nu}-1}^{*}\right) \\
c_{t^{\nu}}^{*}+k_{t^{\nu}+1}^{*} & =f\left(k_{t^{\nu}}^{*}\right)+\phi\left(R_{t^{\nu}}^{*}\right), \text { with } R_{t^{\nu}}^{*}>0
\end{aligned}
$$

Hence

$$
\forall \nu, f^{\prime}\left(k_{t^{\nu}}^{*}\right)=\frac{u^{\prime}\left(c_{t^{\nu}-1}^{*}\right)}{\beta u^{\prime}\left(c_{t^{\nu}}^{*}\right)} \leq \frac{\phi^{\prime}\left(R_{t^{\nu}}^{*}\right)}{\phi^{\prime}(0)}<1 .
$$

Therefore, $\forall \nu, k_{t^{\nu}}^{*}>\widehat{k}$. Observe that there exists $\lambda>0$ such that

$$
\forall \nu, \beta^{t_{\nu}} u^{\prime}\left(c_{t^{\nu}}^{*}\right) \phi^{\prime}\left(R_{t^{\nu}}^{*}\right)=\lambda
$$

This implies $c_{t^{\nu}}^{*} \rightarrow 0$ as $\nu \rightarrow+\infty$. From Lemma $2, k_{t^{\nu}}^{*} \leq A, \forall \nu$. One can suppose $k_{t^{\nu}}^{*} \rightarrow \bar{k} \geq \widehat{k}>0$ and $k_{t^{\nu}+1}^{*} \rightarrow \underline{k}=f(\bar{k})$. From Lemma $3, \underline{k} \in \varphi(\bar{k})$. This implies $c_{t^{\nu}}^{*} \rightarrow \bar{c}=f(\bar{k})-\underline{k}=0$. But, since $\bar{k}>0$, we must have $\bar{c}>0$ (see Le Van and Dana [5]). This contradiction implies the existence of $T_{\infty}$ such that for all $t \geq T_{\infty}$, we have $R_{t}^{*}=0$.

Remark 1 When the function $f$ is concave, the proof will be very short. Indeed, from Le Van and Saglam [6], the sequence $\left(\beta^{t} u^{\prime}\left(c_{t}^{*}\right)\right)_{t}$ satisfies $\sum_{t=0}^{+\infty} \beta^{t} u^{\prime}\left(c_{t}^{*}\right)<$ $+\infty$. Since there exists $\lambda>0$ such that $\beta^{t_{\nu}} u^{\prime}\left(c_{t^{\nu}}^{*}\right) \phi^{\prime}\left(R_{t^{\nu}}^{*}\right)=\lambda$, we have $\beta^{t_{\nu}} u^{\prime}\left(c_{t^{\nu}}^{*}\right) \rightarrow \frac{\lambda}{\phi^{\prime}(0)}>0$. This excludes $\sum_{t=0}^{+\infty} \beta^{t} u^{\prime}\left(c_{t}^{*}\right)$ to be bounded from above. 
In the following corollary, we prove that, even when the initial capital equals 0 , thanks to the natural resource, the country may take-off if the marginal productivity of capital at the origin is larger than the sum of the discount and depreciation rates. But, when this productivity is low, the natural resource cannot prevent the economy to collapse in the long term.

Corollary 1 Let $k_{0} \geq 0$. Assume $\phi^{\prime}(0)<+\infty$.

(a) If $f^{\prime}(0)>\frac{1}{\beta}$, then $k_{t}^{*} \rightarrow k^{s}$ where $k^{s}$ is defined by $f^{\prime}\left(k^{s}\right)=\frac{1}{\beta}$.

(b) If $f$ is concave and $1<f^{\prime}(0) \leq \frac{1}{\beta}$, then $k_{t}^{*} \rightarrow 0$.

Proof: ¿From Proposition 5, we know that $R_{t}^{*}=0$ for $t \geq T_{\infty}$. The optimal sequence $\left(k_{t}^{*}\right)_{t \geq T_{\infty}}$ solves problem $(Q)$ with initial data $k_{T_{\infty}}^{*}>0$. Assertion (a) follows from Dechert and Nishimura [3], while assertion (b) follows, e.g., from Le Van and Dana [5].

We now show that the country may never accumulate in physical capital if the marginal productivity is very low, and the initial capital stock is small.

Proposition 6 Assume $\phi^{\prime}(0)<+\infty$ and $f^{\prime}\left(k_{I}\right)<1$.

(a) Let $k_{0} \geq 0$. Then there exists $T$ with $k_{t}^{*}=0, \forall t \geq T$.

(b) There exists $\varepsilon>0$ such that, if $k_{0} \leq \varepsilon$, then $k_{t}^{*}=0, \forall t$.

Proof: (a) There must be $t_{0}$ with $R_{t_{0}}^{*}>0$. We claim that $R_{t}^{*}>0, \forall t>t_{0}$. Assume $R_{t_{0}+1}^{*}=0$. Then we have the Euler conditions

$$
f^{\prime}\left(k_{t_{0}+1}^{*}\right)=\frac{u^{\prime}\left(c_{t_{0}}^{*}\right)}{\beta u^{\prime}\left(c_{t_{0}+1}^{*}\right)} \geq \frac{\phi^{\prime}(0)}{\phi^{\prime}\left(R_{t_{0}}^{*}\right)}>1
$$

which is impossible. Hence $R_{t_{0}+1}^{*}>0$. By induction, $R_{t}^{*}>0, \forall t>t_{0}$. Thus, for $t \geq t_{0}$, we have the FOC:

$$
f^{\prime}\left(k_{t+1}^{*}\right)=\frac{u^{\prime}\left(c_{t}^{*}\right)}{\beta u^{\prime}\left(c_{t+1}^{*}\right)}=\frac{\phi^{\prime}\left(R_{t+1}^{*}\right)}{\phi^{\prime}\left(R_{t}^{*}\right)}, \text { if } k_{t+1}^{*}>0 .
$$

If there exists an infinite sequence $\left(k_{t_{\nu}+1}^{*}\right)_{\nu}$ with $k_{t_{\nu}+1}^{*}>0, \forall \nu$, then from the previous FOC we have $\lim _{\nu \rightarrow+\infty} f^{\prime}\left(k_{t_{\nu}+1}^{*}\right)=1$ : a contradiction since $\forall \nu, f^{\prime}\left(k_{t_{\nu}+1}^{*}\right) \leq$ $f^{\prime}\left(k_{I}\right)<1$. Therefore, $k_{t}^{*}=0$ for any $t$ large enough.

(b) Consider Problem $(R)$ :

$$
S\left(k_{0}, \bar{S}\right)=\max \sum_{t=0}^{+\infty} \beta^{t} u\left(c_{t}\right)
$$

under the constraints

$$
\begin{aligned}
0 \leq c_{0} & \leq f\left(k_{0}\right)+\phi\left(R_{0}\right) \\
\forall t \geq 1,0 \leq c_{t} & \leq \phi\left(R_{t}\right), 0 \leq R_{t} \\
\sum_{t=0}^{+\infty} R_{t} & \leq \bar{S}
\end{aligned}
$$


We first prove the claim for $k_{0}=0$. Let $\left(R_{t}^{*}, c_{t}^{*}\right)_{t}$ be the solution. We have $\sum_{t=0}^{+\infty} R_{t}^{*}=\bar{S}$ and $c_{t}^{*}=\phi\left(R_{t}^{*}\right), \forall t$. There exists $\lambda$ such that $\forall t, \beta^{t} u^{\prime}\left(\phi\left(R_{t}^{*}\right)\right) \phi^{\prime}\left(R_{t}^{*}\right)=$ $\lambda$. Let $\left(k_{t}, R_{t}\right)_{t}$ be a solution to the initial problem. We have $\sum_{t=0}^{+\infty} R_{t}=\bar{S}$.

Consider

$$
\triangle=\sum_{t=0}^{+\infty} \beta^{t}\left[u\left(\phi\left(R_{t}^{*}\right)\right)-u\left(\phi\left(R_{t}\right)+f\left(k_{t}\right)-k_{t+1}\right)\right] .
$$

We have

$$
\begin{aligned}
\triangle & \geq \sum_{t=0}^{+\infty} \beta^{t} u^{\prime}\left(\phi\left(R_{t}^{*}\right)\right) \phi^{\prime}\left(R_{t}^{*}\right)\left(R_{t}^{*}-R_{t}\right)+\sum_{t=0}^{+\infty} \beta^{t} u^{\prime}\left(\phi\left(R_{t}^{*}\right)\right)\left(k_{t+1}-f\left(k_{t}\right)\right) \\
& \geq \lambda\left(\sum_{t=0}^{+\infty} R_{t}^{*}-\sum_{t=0}^{+\infty} R_{t}\right)+\sum_{t=0}^{+\infty} \beta^{t} u^{\prime}\left(\phi\left(R_{t}^{*}\right)\right)\left(k_{t+1}-f\left(k_{t}\right)\right) \\
& \geq \sum_{t=0}^{+\infty} \beta^{t} u^{\prime}\left(\phi\left(R_{t}^{*}\right)\right)\left(k_{t+1}-f\left(k_{t}\right)\right) .
\end{aligned}
$$

Recall that $\forall t, R_{t+1}^{*}<R_{t}^{*}$. Since $u^{\prime}\left(\phi\left(R_{t}^{*}\right)\right) \phi^{\prime}\left(R_{t}^{*}\right)=\beta u^{\prime}\left(\phi\left(R_{t+1}^{*}\right)\right) \phi^{\prime}\left(R_{t+1}^{*}\right)$ we have $u^{\prime}\left(\phi\left(R_{t}^{*}\right)\right)>\beta u^{\prime}\left(\phi\left(R_{t+1}^{*}\right)\right), \forall t$. From part (a), there exists $T$ such that $k_{t}=0, \forall t \geq T+1$. Therefore

$$
\triangle \geq \sum_{t=1}^{T} \beta^{t} u^{\prime}\left(\phi\left(R_{t}^{*}\right)\right)\left(k_{t}-f\left(k_{t}\right)\right) .
$$

Since $f^{\prime}(k)<1$ and $f(0)=0$, we have $f(k)<k$. Thus, $\triangle>0$. This is a contradiction.

Now, let $k_{0}>0$. Then we have $\beta^{t} u^{\prime}\left(\phi\left(R_{t}^{*}\right)\right) \phi^{\prime}\left(R_{t}^{*}\right)=\lambda$ for some $\lambda>0$ and $t \geq 1$, and $u^{\prime}\left(\phi\left(R_{0}^{*}\right)+f\left(k_{0}\right)\right) \phi^{\prime}\left(R_{0}^{*}\right) \leq \beta u^{\prime}\left(\phi\left(R_{1}^{*}\right)\right) \phi^{\prime}\left(R_{1}^{*}\right)$ with equality if $R_{0}^{*}>0$. The same computations as above give

$$
\triangle \geq \sum_{t=2}^{T} \beta^{t} u^{\prime}\left(\phi\left(R_{t}^{*}\right)\right)\left(k_{t}-f\left(k_{t}\right)\right)+u^{\prime}\left(f\left(k_{0}\right)+\phi\left(R_{0}^{*}\right)\right) k_{1}-\beta u^{\prime}\left(\phi\left(R_{1}^{*}\right)\right) f\left(k_{1}\right) .
$$

When $k_{0}=0$ we have $u^{\prime}\left(\phi\left(R_{0}^{*}\right)\right)>\beta u^{\prime}\left(\phi\left(R_{1}^{*}\right)\right)$. But $R_{0}^{*}$ and $R_{1}^{*}$ are continuous in $k_{0}$. Hence, when $k_{0} \leq \varepsilon$ with $\varepsilon$ small enough, we still have $u^{\prime}\left(f\left(k_{0}\right)+\phi\left(R_{0}^{*}\right)\right)>$ $\beta u^{\prime}\left(\phi\left(R_{1}^{*}\right)\right)$. A contradiction arises as before.

We expect that, when the marginal productivity of capital is low, the country will never accumulate if the stock of non-renewable resource is very large. The result is true if we assume $\widehat{R}=+\infty$. To simplify the proof we will use some explicit forms of the functions $u$ and $\phi$.

Proposition 7 Assume $\phi^{\prime}(0)<+\infty$ and $f^{\prime}\left(k_{I}\right)<1$. Assume also $\widehat{R}=+\infty$, $\phi(R)=a R, a>0$ and $u(c)=\frac{c^{\theta}}{\theta}$ with $0<\theta<1$. Let $k_{0}>0$ be given. Then we have $k_{t}^{*}=0, \forall t$, when $\bar{S}$ is large enough. 
Proof: Let $\left(R_{t}^{*}\right)_{t=0, \ldots,+\infty}$ be the solution. We have

$$
\begin{aligned}
\forall t \geq 1, \mu_{0}+a\left(f\left(k_{0}\right)+a R_{0}^{*}\right)^{\theta-1} & =\beta^{t} a\left(a R_{t}^{*}\right)^{\theta-1} \\
\text { where } \mu_{0} \geq 0, \text { and } \mu_{0} R_{0}^{*} & =0 .
\end{aligned}
$$

We obtain

$$
\begin{aligned}
\forall t \geq 1, R_{t}^{*} & =\frac{\beta^{(1-\theta) t}}{a}\left[\frac{\mu_{0}+a\left(f\left(k_{0}\right)+a R_{0}^{*}\right)^{\theta-1}}{a}\right]^{\frac{1}{\theta-1}} \\
\sum_{t=0}^{+\infty} R_{t}^{*} & =R_{0}^{*}+\frac{\beta^{1-\theta}}{a\left(1-\beta^{1-\theta}\right)}\left[\frac{\mu_{0}+a\left(f\left(k_{0}\right)+a R_{0}^{*}\right)^{\theta-1}}{a}\right]^{\frac{1}{\theta-1}}, \\
\text { and } \mu_{0} R_{0}^{*} & =0 .
\end{aligned}
$$

$R_{0}^{*}$, and when $R_{0}^{*}=0, \mu_{0}$ will be determined by the contraint $\bar{S}=\sum_{t=0}^{+\infty} R_{t}^{*}$. It is obvious that $\mu_{0}$ is a decreasing function of $\bar{S}$, while $R_{0}^{*}$ is an increasing function of $\bar{S}$. Thus, when $\bar{S}$ is high enough, we have $\mu_{0}=0$ and $R_{0}^{*}>0$. Hence, from relation (3), we get $\left(f\left(k_{0}\right)+a R_{0}^{*}\right)^{\theta-1}=\beta\left(a R_{1}^{*}\right)^{\theta-1}$, i.e. $u^{\prime}\left(f\left(k_{0}\right)+a R_{0}^{*}\right)=$ $\beta u^{\prime}\left(a R_{1}^{*}\right)$. Using the proof of Proposition 6 , we conclude that the optimal path $\left(k_{t}^{*}\right)$ equals 0 .

We now study the long term of our economy with an exhaustible resource. Obviously, in the long term, the exhaustible resource is depleted.

We know, from Dechert and Nishimura [3], that if $f^{\prime}(0)<\frac{1}{\beta}<\max \left\{\frac{f(k)}{k}\right.$ : $k>0\}$, then there exists $k^{c}\left(<k_{I}<\widetilde{k}\right.$, with $\left.f^{\prime}(\widetilde{k})=1\right)$, such that if $k_{0}<k^{c}$ then any solution $\mathbf{k}$ to Problem $(Q)$ converges to 0 , and if $k_{0}>k^{c}$, then it converges to a high steady state $k^{s}$ fulfilling $f^{\prime}\left(k^{s}\right)=\frac{1}{\beta}$. In other words, we have a poverty trap. We will show, under some more assumptions, that if $\bar{S}$ is high enough the poverty trap can be passed over in our model. We need a preliminary lemma.

Lemma 4 Consider the following problem:

$$
\max \sum_{t=0}^{+\infty} \beta^{t} u\left(c_{t}\right)
$$

under the constraints

$$
\begin{aligned}
c_{0}+k_{1} & \leq f\left(k_{0}\right) \\
c_{1}+k_{2} & \leq f\left(k_{1}\right)+a, a \geq 0, \\
c_{t}+k_{t+1} & \leq f\left(k_{t}\right) \\
\forall t, 0 \leq c_{t}, 0 & \leq k_{t}, k_{0}>k_{I} .
\end{aligned}
$$


Assume $f^{\prime}\left(k_{I}\right)=+\infty$. Then, for any $a \geq 0$, we have a unique solution $\left\{k_{1}^{*}(a), \ldots, k_{t}^{*}(a), \ldots.\right\}$. Moreover, $k_{1}^{*}(a)>k_{I}, k_{1}^{*}(a)$ decreases while $f\left(k_{1}^{*}(a)\right)+a$ increases, when a increases.

Proof: Under the assumption that $f^{\prime}(0)<\frac{1}{\beta}<\max \left\{\frac{f(k)}{k}: k>0\right\}$, when $k_{0}>k_{I}$ and $a=0$, any optimal path will be bounded below by $k_{I}$ (see Dechert and Nishimura [3]). We just consider the feasible paths which are bounded below by $k_{I}$. In this case, the constraints will be strictly concave, since the function $f$ is strictly concave for $k>k_{I}$. Hence the solution will be unique. Moreover the Value function is strictly concave and differentiable when $a=0$.

Since for $a=0, k_{1}^{*}(a)>k_{I}, f\left(k_{1}^{*}(a)\right)>k_{I}$, it will still be true when $a>0$ small enough. We have the Bellman equation

$$
V\left(f\left(k_{0}\right)\right)=\max _{0 \leq y \leq f\left(k_{0}\right)}\left\{u\left(f\left(k_{0}\right)-y\right)+\beta V(f(y)+a)\right\}
$$

When $a>0$ is small, $k_{1}^{*}(a)$ satisfies

$$
\left.u^{\prime}\left(f\left(k_{0}\right)-k_{1}^{*}(a)\right)=\beta V^{\prime}\left(f\left(k_{1}^{*}(a)\right)\right)+a\right) f^{\prime}\left(k_{1}^{*}(a)\right) .
$$

Assume that $a$ increases and $f\left(k_{1}^{*}(a)\right)+a$ decreases. In this case, $k_{1}^{*}(a)$ decreases, and the left-hand side of (5) decreases. But the right-hand side increases since $V^{\prime}(k)$ and $f^{\prime}(k)$ are decreasing functions for $k>k_{I}$. We have a contradiction.

It is easy to check that $k_{1}^{*}(a)$ decreases when $a$ is small and increases.

Assume there exists $\bar{a}$, the maximum value of $a$ such that $k_{1}^{*}(a) \geq k_{I}$. Let $a$ converge to $\bar{a}$. In this case, $k_{1}^{*}(a)$ converges to $k_{I}$. For $a<\bar{a}$, we have the Euler condition:

$$
\begin{aligned}
f^{\prime}\left(k_{1}^{*}(a)\right) & =\frac{u^{\prime}\left(f\left(k_{0}\right)-k_{1}^{*}(a)\right)}{\beta u^{\prime}\left(f\left(k_{1}^{*}(a)+a-k_{2}^{*}(a)\right)\right)} \\
& \leq \frac{u^{\prime}\left(f\left(k_{0}\right)-k_{1}^{*}(a)\right)}{\beta u^{\prime}\left(f\left(k_{1}^{*}(a)+a\right)\right)} .
\end{aligned}
$$

Taking the limits we get a contradiction:

$$
+\infty=f^{\prime}\left(k_{I}\right) \leq \frac{u^{\prime}\left(f\left(k_{0}\right)-k_{I}\right)}{\beta u^{\prime}\left(f\left(k_{I}+a\right)\right)}<+\infty .
$$

Hence, for all $a \geq 0, k_{1}^{*}(a)>k_{I}$. We can prove as before, when $a$ is small, that $k_{1}^{*}(a)$ decreases while $f\left(k_{1}^{*}(a)\right)+a$ increases, when $a$ increases.

Proposition 8 Assume there exists $\widetilde{R} \in(0, \widehat{R})$ such that $\phi(R)=a R, a>0$ when $0 \leq R \leq \widetilde{R}$, and $1<f^{\prime}(0) \leq \frac{1}{\beta} \leq \max \left\{\frac{f(k)}{k}: k>0\right\}$. Define $k_{0}^{\prime}$ by $f\left(k_{0}^{\prime}\right)=\phi(\widetilde{R})$. Assume $k_{0}^{\prime}>k_{I}$. Assume moreover that $f^{\prime}\left(k_{I}\right)=+\infty$. Let $k_{0} \geq 0$. The optimal sequence, $k_{t}^{*} \rightarrow k^{s}$ as $t \rightarrow+\infty$, if $\bar{S}$ is high enough. 
Proof: ¿From Proposition 5, there exists $T_{\infty}$ such that:

$$
\begin{aligned}
c_{T_{\infty}-2}^{*}+k_{T_{\infty}-1}^{*} & =f\left(k_{T_{\infty}-2}^{*}\right)+\phi\left(R_{T_{\infty}-2}^{*}\right) \\
c_{T_{\infty}-1}^{*}+k_{T_{\infty}}^{*} & =f\left(k_{T_{\infty}-1}^{*}\right)+\phi\left(R_{T_{\infty}-1}^{*}\right) \\
c_{T_{\infty}}^{*}+k_{T_{\infty}+1}^{*} & =f\left(k_{T_{\infty}}^{*}\right) \\
c_{t}^{*}+k_{t+1}^{*} & =f\left(k_{t}^{*}\right), \forall t \geq T_{\infty}+1 .
\end{aligned}
$$

Case 1: We have $R_{T_{\infty}-1}^{*} \geq \widetilde{R}$.

Let $k_{0}^{* \prime}$ satisfy $f\left(k_{0}^{* \prime}\right)=f\left(k_{T_{\infty}-1}^{*}\right)+\phi\left(R_{T_{\infty}-1}^{*}\right)$. Then, $k_{0}^{* \prime}>k_{I}$. The sequence $\left\{k_{t}^{*}\right\}_{t \geq T_{\infty}}$ is optimal for the growth model with initial capital $k_{0}^{* \prime}>k_{I}$. It will converge to the steady state $k^{s}$ since $k_{I}>k^{c}$.

Case 2: We have $R_{T_{\infty}-1}^{*} \leq \widetilde{R}$ and $R_{T_{\infty}-2}^{*} \leq \widetilde{R}$.

We have, from the Euler conditions

$$
f^{\prime}\left(k_{T_{\infty}-1}^{*}\right) \leq \frac{\phi^{\prime}\left(R_{T_{\infty}-1}^{*}\right)}{\phi^{\prime}\left(R_{T_{\infty}-2}^{*}\right)}=1
$$

hence, $k_{T_{\infty}-1}^{*} \geq \widetilde{k}>k_{I}\left(f^{\prime}(\widetilde{k})=1\right)$ and as before, the optimal path converges to the steady state.

Case 3: We have $R_{T_{\infty}-1}^{*} \leq \widetilde{R}$ and $R_{T_{\infty}-2}^{*} \geq \widetilde{R}$.

Let $k_{0}^{* \prime}$ satisfy $f\left(k_{0}^{* \prime}\right)=f\left(k_{T_{\infty}-2}^{*}\right)+\phi\left(R_{T_{\infty}-2}^{*}\right)$. Then, $k_{0}^{* \prime}>k_{I}$. From Lemma 4 , we have $k_{T_{\infty}-1}^{*}>k_{I}$. As before, the optimal path converges to the steady state.

In the following proposition we drop the assumption that the technology $\phi$ is linear in a neighborhood of 0 . But we will strengthen the assumptions on the marginal productivity of capital.

Proposition 9 Assume there exists $\widetilde{R} \in(0, \widehat{R})$ such that, if $k_{0}^{\prime}$ satisfies $f\left(k_{0}^{\prime}\right)=$ $\phi(\widetilde{R})$, then $k_{0}^{\prime}>k_{I}$. Assume moreover that $f^{\prime}\left(k_{I}\right)=+\infty$ and $\frac{\phi^{\prime}(0)}{\phi^{\prime}(\widetilde{R})}<f^{\prime}(0) \leq$ $\frac{1}{\beta} \leq \max \left\{\frac{f(k)}{k}: k>0\right\}$. Let $k_{0} \geq 0$. The optimal sequence, $k_{t}^{*} \rightarrow k^{s}$ as $t \rightarrow+\infty$, if $\bar{S}$ is high enough.

Proof: We just consider Case 2 in the proof of the previous Proposition : $R_{T_{\infty}-1}^{*} \leq \widetilde{R}$ and $R_{T_{\infty}-2}^{*} \leq \widetilde{R}$.

We have, from the Euler conditions

$$
f^{\prime}\left(k_{T_{\infty}-1}^{*}\right) \leq \frac{\phi^{\prime}\left(R_{T_{\infty}-1}^{*}\right)}{\phi^{\prime}\left(R_{T_{\infty}-2}^{*}\right)} \leq \frac{\phi^{\prime}(0)}{\phi^{\prime}(\widetilde{R})}<f^{\prime}(0) .
$$

Observe that $f^{\prime}(k)>f^{\prime}(0)$ for $k \in\left[0, k^{s}\right]$. Hence $k_{T_{\infty}-1}^{*}>k^{s}>k_{I}$. The optimal sequence $\left\{k_{t}^{*}\right\}_{t \geq T_{\infty}}$ converges therefore to $k^{s}$.

In the following proposition, we assume $\widetilde{R}=+\infty$. 
Proposition 10 Assume $\widehat{R}=+\infty, \phi(R)=a R, a>0$ and $1<f^{\prime}(0) \leq \frac{1}{\beta} \leq$ $\max \left\{\frac{f(k)}{k}: k>0\right\}$. Let $k_{0} \geq 0$. The optimal sequence, $k_{t}^{*} \rightarrow k^{s}$ as $t \rightarrow+\infty$, if $\bar{S}$ is high enough.

Proof: Let $\left(\widehat{S}^{\nu}\right)$ be a sequence which converges to $+\infty$. We consider two cases. Case 1: For any $\nu$, the optimal sequence $\left(R_{t}^{* \nu}\right)$ verifies, $R_{0}^{* \nu}=\bar{S}^{\nu}$. Let $k_{0}^{\nu}$ satisfy $f\left(k_{0}^{\nu}\right)=f\left(k_{0}\right)+a \bar{S}^{\nu}$. For $\nu$ large enough, $k_{0}^{\nu}>k^{c}$. An optimal sequence $\left(k_{t}^{* \nu}\right)$ is also an optimal sequence for the convex-concave optimal growth-model with initial endowment equal to $k_{0}^{\nu}$. Since this one is larger than the critical value $k^{c}$, the optimal path $\left(k_{t}^{* \nu}\right)$ will converge to the high steady state $k^{s}$. And the proof is over.

Case 2. From Proposition 5, there exists $T_{\infty}$ such that:

$$
\begin{aligned}
c_{T_{\infty}-2}^{*}+k_{T_{\infty}-1}^{*} & =f\left(k_{T_{\infty}-2}^{*}\right)+a R_{T_{\infty}-2}^{*} \\
c_{T_{\infty}-1}^{*}+k_{T_{\infty}}^{*} & =f\left(k_{T_{\infty}-1}^{*}\right)+a R_{T_{\infty}-1}^{*} \\
c_{T_{\infty}}^{*}+k_{T_{\infty}+1}^{*} & =f\left(k_{T_{\infty}}^{*}\right) \\
c_{t}^{*}+k_{t+1}^{*} & =f\left(k_{t}^{*}\right), \forall t \geq T_{\infty}+1 .
\end{aligned}
$$

We have

$$
f^{\prime}\left(k_{T_{\infty}-1}^{*}\right) \leq \frac{u^{\prime}\left(c_{T_{\infty}-2}^{*}\right)}{\beta u^{\prime}\left(c_{T_{\infty}-1}^{*}\right)} \leq \frac{\phi^{\prime}\left(R_{T_{\infty}-1}^{*}\right)}{\phi^{\prime}\left(R_{T_{\infty}-2}^{*}\right)}=1 .
$$

We cannot have $k_{T_{\infty}-1}^{*}=0$, since $f^{\prime}(0)>1$. Hence $k_{T_{\infty}-1}^{*} \geq \widetilde{k}$ which is the unique point with $f^{\prime}(\widetilde{k})=1$. Moreover, $\widetilde{k}>k^{s}>k^{c}$. Let $k_{0}^{\prime}$ satisfy $f\left(k_{0}^{\prime}\right)=f\left(k_{T_{\infty}-1}^{*}\right)+a R_{T_{\infty}-1}^{*}$. Then, $k_{0}^{\prime}>k_{T_{\infty}-1}^{*}>k^{s}>k^{c}$. The sequence $\left(k_{t}^{*}\right), t=T_{\infty}-1, \ldots,+\infty$ is also an optimal solution to the convex-concave optimal growth-model with initial endowment equal to $k_{0}^{\prime}$. Since $k_{0}^{\prime}>k^{c}$, the path $\left(k_{t}^{*}\right), t=T_{\infty}-1, \ldots,+\infty$ converges to the high steady state.

Remark 2 Proposition 10 shows that the overshooting effect exists when $\bar{S}$ is large enough. Indeed, if we are in Case 1 of the proof, then when $\bar{S}$ is large, the value $k_{0}^{\prime}$ defined by $f\left(k_{0}^{\prime}\right)=f\left(k_{0}\right)+\phi(\bar{S})$ will be larger than $k^{s}$. From Dechert and Nishimura [3], the optimal path $\left(k_{t}^{*}\right)$ will be decreasing and converges to $k^{s}$. If we are in Case 2, then, from the proof, there exists $k_{T_{\infty}-1}^{*}>k^{s}$.

Remark $\mathbf{3}$ We will make a small change in $\mathbf{H 2}$ by dropping the assumption $f^{\prime}(+\infty)<1$. Assume $f(k)=A k, A>1$ and $\phi(R)=a R$. Then it is optimal to exhaust the resource at period 0 . Indeed, the Euler conditions will be:

$$
\begin{aligned}
A=f^{\prime}\left(k_{t+1}^{*}\right) & \leq \frac{\phi^{\prime}\left(R_{t+1}^{*}\right)}{\phi^{\prime}\left(R_{t}^{*}\right)} \\
\text { if } R_{t+1}^{*}>0 & \\
\text { and } A=f^{\prime}\left(k_{t+1}^{*}\right) & \geq \frac{\phi^{\prime}(0)}{\phi^{\prime}\left(R_{t}^{*}\right)}=1 \\
\text { if } R_{t+1}^{*}=0 . &
\end{aligned}
$$


¿From these inequalities, since $A>1$, we cannot have $R_{t+1}^{*}>0$, for some $t \geq 0$

since $\frac{\phi^{\prime}\left(R_{t+1}^{*}\right)}{\phi^{\prime}\left(R_{t}^{*}\right)}=1$. Hence $R_{t+1}^{*}=0, \forall t \geq 0$, and $R_{1}^{*}=\bar{S}$. Observe that $k_{t}^{*} \rightarrow+\infty$ if $A>\frac{1}{\beta}$.

However, it seems not realistic to completely exhaust the resource in one period. Actually, in our discrete time model, one period may be many years. Our story tells that, in developed countries which possess non-renewable resources, it is optimal to exhaust it quickly in order to build a large initial capital stock and then converge faster to the steady state.

\section{Competitive Equilibrium With Tax/Subsidy}

In our intertemporal economy, there is a single firm which produces, with a technology represented by the function $f$, an aggregate good which can be consumed or used as physical capital. This firm also imports this aggregate good. The importations are covered by the exportation of the natural resource that the firm extracts. Since we face increasing returns for low levels of capital, we will define an equilibrium with a tax/subsidy scheme to firms, which will be supported by the consumer or redistributed to her as a lump sum transfer. The scheme is defined as follows. If the value of the input is larger than the value of the output, then firms will receive a subsidy equal to the difference. A tax will be defined analogously in the reverse case.

Let $\left\{k_{t}\right\}_{t=1,2, . .,+\infty}$ be a firm production plan. Formally, if $\left(p_{t}\right)$ is the sequence of prices of the aggregate good, $r$ is the price of initial capital stock $k_{0}$, and $\left\{\sigma_{t}(.)\right\}_{t}$ is a system of subsidy, then firm solves the problem

$$
\Pi=\max _{\left(k_{t}\right),\left(R_{t}\right)}\left[\sum_{t=0}^{+\infty} p_{t}\left(f\left(k_{t}\right)-k_{t+1}\right)-r k_{0}+\sum_{t=0}^{+\infty} \sigma_{t}\left(k_{t}\right)+\sum_{t=0}^{+\infty} p_{t} \phi\left(R_{t}\right)\right]
$$

considering the tax/subsidy scheme as given, and under the constraints:

$$
\begin{aligned}
\forall t, 0 \leq k_{t+1} & \leq f\left(k_{t}\right)+\phi\left(R_{t}\right), 0 \leq R_{t} \\
\sum_{t=0}^{+\infty} R_{t} & \leq \bar{S}, \text { and } k_{0} \text { is given. }
\end{aligned}
$$

There is one representative consumer who owns the firm and the initial capital stock $k_{0}$. Her problem is

$$
\max _{\left(c_{t}\right)} \sum_{t=0}^{+\infty} \beta^{t} u\left(c_{t}\right)
$$

under the constraints

$$
\sum_{t=0}^{+\infty} p_{t} c_{t} \leq \Pi+r k_{0}-\sum_{t=0}^{+\infty} \sigma_{t}\left(k_{t}\right)
$$


and $c_{t} \geq 0, \forall t$.

Let us recall that $l^{\infty}=\left\{\mathbf{x}: \sup _{t}\left|x_{t}\right|<+\infty\right\}$ and $l^{1}=\left\{\mathbf{p}: \sum_{t=0}^{+\infty}\left|p_{t}\right|<+\infty\right\}$.

Definition 1 The list $\left\{\mathbf{c}^{*}, \mathbf{k}^{*}, \mathbf{R}^{*}, \mathbf{p}^{*}, r^{*}, \boldsymbol{\sigma}^{*}().\right\}$, is a competitive equilibrium of our economy if:

(1) $\mathbf{c}^{*} \in l_{+}^{\infty}, \mathbf{k}^{*} \in l_{+}^{\infty}, \mathbf{R}^{*} \in l_{+}^{\infty}, \mathbf{p}^{*} \in l_{+}^{1}, r^{*} \geq 0,\left\{\mathbf{p}^{*}, r^{*}\right\} \neq\{\mathbf{0}, 0\}$.

(2) Given $\left\{\mathbf{p}^{*}, r^{*}, \boldsymbol{\sigma}^{*}().\right\},\left\{\mathbf{k}^{*}, \mathbf{R}^{*}\right\}$ solve the problem of the firm, i.e.

$$
\begin{aligned}
\Pi^{*} & =\max _{\left(k_{t}\right),\left(R_{t}\right)}\left[\sum_{t=0}^{+\infty} p_{t}^{*}\left(f\left(k_{t}\right)-k_{t+1}\right)-r^{*} k_{0}+\sum_{t=0}^{+\infty} \sigma_{t}^{*}\left(k_{t}\right)+\sum_{t=0}^{+\infty} p_{t}^{*} \phi\left(R_{t}\right)\right] \\
& =\left[\sum_{t=0}^{+\infty} p_{t}^{*}\left(f\left(k_{t}^{*}\right)-k_{t+1}^{*}\right)-r^{*} k_{0}+\sum_{t=0}^{+\infty} \sigma_{t}^{*}\left(k_{t}^{*}\right)+\sum_{t=0}^{+\infty} p_{t}^{*} \phi\left(R_{t}^{*}\right)\right] .
\end{aligned}
$$

(3) Given $\left\{\mathbf{p}^{*}, r^{*}, \boldsymbol{\sigma}^{*}().\right\}, \mathbf{c}^{*}$ solve the consumer's problem:

$$
\sum_{t=0}^{+\infty} \beta^{t} u\left(c_{t}^{*}\right)=\max _{\left(c_{t}\right)} \sum_{t=0}^{+\infty} \beta^{t} u\left(c_{t}\right)
$$

under the constraints

$$
\sum_{t=0}^{+\infty} p_{t}^{*} c_{t} \leq \Pi^{*}+r^{*} k_{0}-\sum_{t=0}^{+\infty} \sigma_{t}^{*}\left(k_{t}^{*}\right) .
$$

(4) Market Clearing:

$\forall t, c_{t}^{*}+k_{t+1}^{*}=f\left(k_{t}^{*}\right)+\phi\left(R_{t}^{*}\right)$,

Proposition 11 Assume H1, H2, H3, $\phi^{\prime}(0)>1$ and $k_{0}>0$. Moreover, we assume

(i) either $f^{\prime}(0)>\frac{1}{\beta}$,

(ii) or the assumptions in Proposition 8 or Proposition 9 are fulfilled.

Let $\left\{\mathbf{c}^{*}, \mathbf{k}^{*}, \mathbf{R}^{*}\right\}$ be a solution to our problem $(\mathrm{P})$. Define:

$$
\begin{aligned}
\forall t, p_{t}^{*} & =\beta^{t} u^{\prime}\left(c_{t}^{*}\right), r^{*}=p_{0}^{*} f^{\prime}\left(k_{0}^{*}\right), \\
\text { and } \sigma_{t}^{*}(k) & =p_{t}^{*} f^{\prime}\left(k_{t}^{*}\right) k-p_{t}^{*} f(k) .
\end{aligned}
$$

Then the list $\left\{\mathbf{c}^{*}, \mathbf{k}^{*}, \mathbf{R}^{*}, \mathbf{p}^{*}, r^{*}, \boldsymbol{\sigma}^{*}().\right\}$ is a competitive equilibrium.

Moreover, the equilibrium profit of the firm is positive.

Proof: Obviously, $\left\{\mathbf{c}^{*}, \mathbf{k}^{*}, \mathbf{R}^{*}\right\} \in l^{\infty} \times l^{\infty} \times l^{\infty}$. We now prove that $\left\{\left(\beta^{t} u^{\prime}\left(c_{t}^{*}\right)\right)_{t}\right\} \in$ $l^{1}$. Indeed, under our assumptions, the optimal path $\left\{k_{t}^{*}\right\}_{t}$ converges to the high steady state $k^{s}$ and the optimal consumptions converge to $c^{s}=f\left(k^{s}\right)-k^{s}>0$. Hence $\sum_{t=0}^{+\infty} \beta^{t} u^{\prime}\left(c_{t}^{*}\right)<+\infty$. 
We now prove that, given $\left\{\mathbf{p}^{*}, r^{*}, \boldsymbol{\sigma}^{*}().\right\},\left\{\mathbf{k}^{*}, \mathbf{R}^{*}\right\}$, solve the problem of the firm. First, observe that there exists $\lambda>0$ and a non-negative sequence $\boldsymbol{\mu}$ such that

$$
\forall t, p_{t}^{*} \phi^{\prime}\left(R_{t}^{*}\right)=\beta^{t} u^{\prime}\left(c_{t}^{*}\right) \phi^{\prime}\left(R_{t}^{*}\right)=\lambda-\mu_{t}, \text { and } \mu_{t} R_{t}^{*}=0,
$$

and from Euler conditions:

$$
\forall t, p_{t}^{*} f^{\prime}\left(k_{t}^{*}\right)=p_{t-1}^{*}
$$

Now, take a feasible sequence from $k_{0},\left(k_{0}, k_{1}, \ldots, k_{t}, \ldots\right)$ and let

$$
\begin{aligned}
\triangle_{T} & =\left[\sum_{t=0}^{T} p_{t}^{*}\left(f\left(k_{t}^{*}\right)-k_{t+1}^{*}\right)-r^{*} k_{0}+\sum_{t=0}^{T} \sigma^{*}\left(k_{t}^{*}\right)+\sum_{t=0}^{T} p_{t}^{*} \phi\left(R_{t}^{*}\right)\right] \\
& -\left[\sum_{t=0}^{T} p_{t}^{*}\left(f\left(k_{t}\right)-k_{t+1}\right)-r^{*} k_{0}+\sum_{t=0}^{T} \sigma^{*}\left(k_{t}\right)+\sum_{t=0}^{T} p_{t}^{*} \phi\left(R_{t}\right)\right] .
\end{aligned}
$$

Then we have:

$$
\begin{aligned}
\triangle_{T} & \geq \sum_{t=1}^{T}\left[\left(p_{t}^{*} f^{\prime}\left(k_{t}^{*}\right)-p_{t-1}^{*}\right)\left(k_{t}^{*}-k_{t}\right)\right]-p_{T}^{*}\left(k_{T+1}^{*}-k_{T+1}\right)+\sum_{t=0}^{T} p_{t}^{*} \phi^{\prime}\left(R_{t}^{*}\right)\left(R_{t}^{*}-R_{t}\right) \\
& =-p_{T}^{*}\left(k_{T+1}^{*}-k_{T+1}\right)+\lambda \sum_{t=0}^{T}\left(R_{t}^{*}-R_{t}\right)-\sum_{t=0}^{T} \mu_{t} R_{t}^{*}+\sum_{t=0}^{T} \mu_{t} R^{t} \\
& \geq-p_{T}^{*} k_{T+1}^{*}+\lambda \sum_{t=0}^{T}\left(R_{t}^{*}-R_{t}\right) .
\end{aligned}
$$

Since $\sum_{t=0}^{+\infty} R_{t}^{*}=\bar{S} \geq \sum_{t=0}^{+\infty} R_{t}$, we have $\lim _{T} \triangle_{T} \geq \lim _{T}\left\{-p_{T}^{*} k_{T+1}^{*}\right\}$. But $k_{T+1}^{*}$ converges to $k^{s}$. Since $\sum_{t=0}^{+\infty} p_{t}^{*}<+\infty$, we have $p_{t}^{*} \rightarrow 0$. Therefore, $\lim _{T} \triangle_{T} \geq 0$.

We now prove that, given $\left\{\mathbf{p}^{*}, r^{*}, \boldsymbol{\sigma}^{*}().\right\}, \mathbf{c}^{*}$ solves the consumer's problem. Indeed, let c satisfy

$$
\sum_{t=0}^{+\infty} p_{t}^{*} c_{t} \leq \Pi^{*}+r^{*} k_{0}-\sum_{t=0}^{T} \sigma^{*}\left(k_{t}^{*}\right) .
$$

We have

$$
\begin{aligned}
\sum_{t=0}^{+\infty} \beta^{t} u\left(c_{t}^{*}\right)-\sum_{t=0}^{+\infty} \beta^{t} u\left(c_{t}\right) & \geq \sum_{t=0}^{+\infty} \beta^{t} u^{\prime}\left(c_{t}^{*}\right)\left(c_{t}^{*}-c_{t}\right) \\
& =\sum_{t=0}^{+\infty} p_{t}^{*}\left(c_{t}^{*}-c_{t}\right) \geq 0
\end{aligned}
$$

since $\sum_{t=0}^{+\infty} p_{t}^{*} c_{t}^{*}=\Pi^{*}+r^{*} k_{0}-\sum_{t=0}^{T} \sigma^{*}\left(k_{t}^{*}\right)$. 
Finally, market clearing condition is obviously satisfied.

To prove that the profit, at equilibrium, of the firm is positive, observe that $\Pi^{*} \geq\left[\sum_{t=0}^{+\infty} p_{t}^{*}\left(f\left(k_{t}\right)-k_{t+1}\right)-r^{*} k_{0}\right]+\sum_{t=0}^{+\infty} \sigma_{t}^{*}\left(k_{t}\right)+\sum_{t=0}^{+\infty} p_{t}^{*} \phi\left(R_{t}\right)$ for any feasible sequences $\left(k_{0}, k_{1}, \ldots, k_{t}, \ldots\right),\left(R_{0}, R_{1}, \ldots, R_{t}, \ldots\right)$. The sequences $\left(k_{0}, 0, \ldots, 0, ..\right)$ and $\left(R_{t}^{*}\right)_{t=10, . .,+\infty}$ are feasible. Therefore,

$$
\Pi^{*} \geq p_{0}^{*} f\left(k_{0}\right)-r^{*} k_{0}+\left[p_{0}^{*} f^{\prime}\left(k_{0}\right)-p_{0}^{*} f\left(k_{0}\right)\right]+\sum_{t=0}^{+\infty} p_{t}^{*} \phi\left(R_{t}^{*}\right)>0
$$

since $p_{0}^{*} f\left(k_{0}\right)-r^{*} k_{0}+\left[p_{0}^{*} f^{\prime}\left(k_{0}\right)-p_{0}^{*} f\left(k_{0}\right)\right]=0$, from the very definition of $r^{*}$.

\section{Summary of the main results and conclusion}

We summarize below the main results, according to the characteristics of the technology.

(a) High marginal productivity of capital

Assume $\phi^{\prime}(0)<+\infty$ and $F^{\prime}(0)>\rho+\delta$. Then the optimal capital path $\left(k_{t}^{*}\right)$ converges to the high steady state $k^{s}$, with $F^{\prime}\left(k^{s}\right)=\rho+\delta$. The stock of non-renewable resource is exhausted in finite time.

(b) Intermediate marginal productivity of capital

Assume $\phi^{\prime}(0)<+\infty$ and $\delta<F^{\prime}(0) \leq \rho+\delta \leq \max \left\{\frac{F(k)}{k}+1-\delta: k>0\right\}$. The stock of non-renewable resource is exhausted in finite time.

(b.1) If the production function $F$ is concave, then the optimal capital path converges to zero.

(b.2) If the production function is convex-concave, the revenue function is linear (i.e. the price is inelastic with respect to the demand) in a neighborhood of the origin, the initial resource stock is large enough and $F^{\prime}\left(k_{I}\right)=+\infty$, then the optimal capital stock converges to the high steady state $k^{s}$.

(b.3) We drop the assumption that $\phi$ is linear in a neighborhood of the origin. We maintain the conditions that the initial resource stock is large and $F^{\prime}\left(k_{I}\right)=+\infty$. If the maximum of the revenue function $\widehat{R}$ is large and the marginal productivity of capital at the origin $F^{\prime}(0)$ is high enough, but less than the sum of the social discount rate $\rho$ nd the depreciation rate $\delta$, then the optimal capital stock converges to the high steady state $k^{s}$.

(c) Low marginal productivity of capital

Assume $\phi^{\prime}(0)<+\infty$ and $F^{\prime}(k)<\delta, \forall k$.

(c.1) There exists $T$ such that $k_{t}^{*}=0, \forall t>T$.

(c.2) The optimal capital path vanishes for any $k_{0}$ small enough. 
(c.3) Assume that the revenue function is linear and the utility function CRRA. Given $k_{0}$, the optimal capital path vanishes when the initial resource stock $\bar{S}$ is large.

We have then shown that exhaustible resources can, under certain circumstances among which the most important is a high marginal productivity of capital at the origin compared with the social discount rate, allow a developing economy to escape from the poverty trap. The initial abundance of the resource and the value of the maximum marginal revenue that can be obtained by selling abroad the resource extracted are also determining. If the initial resource stock is large and if it is possible to extract a large amount of resource immediately, it is possible to escape from the poverty trap even if the marginal productivity of capital at the origin is intermediate. On the contrary, in the same case but with a low marginal productivity of capital, the economy consumes first its resource and then collapses when it it exhausted.

Finally, we prove, by using a tax/subsidy scheme for firms, that any optimal path may be decentralized in a competitive equilibrium.

\section{References}

[1] Azariadis, C. and Stachurski, J. (2005), Poverty Traps in Handbook of Economic Growth, P. Aghion and S. Durlauf eds., North Holland.

[2] Dasgupta, P. and Heal, G. (1974), The Optimal Depletion of Exhaustible Resources, Review of Economic Studies, 41, 3-28.

[3] Dechert, W.D. and Nishimura, K. (1983), A complete characterization of optimal growth paths in an aggregate model with a non-concave production function, Journal of Economic Theory, 31, 332-354.

[4] Eliasson, L. and Turnowsky S. (2004), Renewable resources in an endogenously growin economy: balanced growth and transitional dynamics, Journal of Environmental Economics and Management, 48, 1018-1049.

[5] Le Van, C. and R.A. Dana (2003), Dynamic Programming in Economics Kluwer Academic Publishers.

[6] Le Van C. and Saglam, H.C. (2004), Optimal growth models and the Lagrange multiplier, Journal of Mathematical Economics, 40, 393-410. 